\title{
Profilaxis de neumonía por Pneumocystis jiroveci en niños y adultos sometidos a trasplante de órganos sólidos y de precursores hematopoyéticos
}

\author{
M. Pilar Gambra y Teresa Bidart
}

\section{Prophylaxis against Pneumocystis pneumonia in pediatric and adult patients undergoing solid organ or hematopoietic stem cells transplantation}

Pneumocystis jiroveci is an important pathogen in patients undergoing SOT and HSCT. Universal prophylaxis is recommended for all adults and children with SOT and HSCT, considering that its use significantly reduces the occurrence and mortality associated with pneumonia by this agent. The drug of choice is cotrimoxazole (A1) three times a week, low-dose scheme, that has proved equally effective and better tolerated than the daily regimen and/or at high doses. Prophylaxis starts 7 to 14 days post transplant in SOT recipients and post-implant in HSCT, with an average duration of 6 months except in liver and lung transplant as in HSCT with significant degree of immunosuppression, that lasts for 1 year. Alternatives for prophylaxis are dapsone (B2), aerosolized pentamidine (B2) and atovaquone (C2).

Key words: Pneumocystis jiroveci, Pneumocystis pneumonia, prophylaxis, sulpha-trimethoprim, dapsone, pentamidine, transplant, solid organ transplantation, hematopoietic stem cells transplantation.

Palabras clave: Pneumocystis jiroveci, neumonía por Pneumocystis, profilaxis, cotrimoxazol, dapsona, pentamidina, trasplante, trasplante de órganos sólidos, trasplante de precursores hematopoyéticos.

\section{Introducción}

$P$ neumocystis jiroveci es un patógeno importante en pacientes sometidos a trasplante de órganos sólidos (TOS) y trasplante de precursores hematopoyéticos (TPH). La enfermedad resulta de reactivación de una infección latente y de su adquisición a través de fuentes medio-ambientales. Al igual que en el resto de los agentes infecciosos que afectan a los pacientes inmunocomprometidos, el riesgo de enfermedad depende de la intensidad y duración del compromiso del sistema inmune así como de la condición médica subyacente. La profilaxis con cotrimoxazol ha generado una importante disminución de incidencia de enfermedad por $P$. jiroveci, Toxoplasma gondii, Isospora belli, Nocardia asteroides, Listeria monocytogenes, infecciones urinarias, infecciones respiratorias e infecciones digestivas. Lamentablemente su uso va apareado a problemas de tolerancia y toxicidad.

\section{Epidemiología}

\section{Trasplante de órganos sólidos}

Las cifras de incidencia varían en la literatura científica para cada órgano trasplantado. En términos generales, es mayor en trasplante de corazón y corazón-pulmón y menor en el trasplante renal. Al mismo tiempo, la incidencia es claramente menor en pacientes que reciben quimioprofilaxis (QP) apropiada.
Una cohorte retrospectiva identificó 25 casos de enfermedad por $P$. jiroveci en 1.299 pacientes sometidos a TOS. A pesar de que la profilaxis no fue uniforme en tiempo ni en dosis, la mayoría de los pacientes la recibió. La mayor incidencia fue observada en trasplante de corazón y corazón-pulmón (5-22 /1.000 personas año), seguida por trasplante de hígado (10/1.000 personas año) y la menor incidencia se observó en trasplante renal $(0,8 / 1.000 \text { personas año })^{1}$.

En otra cohorte retrospectiva, de 1.192 pacientes sometidos a trasplantes renales sin profilaxis, la incidencia de neumonía por $P$. jiroveci varió de 0,6 a 14,3\% dependiendo del protocolo inmunosupresor ${ }^{2}$. Esta cifra en pacientes con QP disminuyó a $0,4 \%$ en una cohorte retrospectiva reciente ${ }^{3}$.

\section{Trasplante de precursores hematopoyéticos}

La incidencia de neumonía por $P$. jiroveci en TPH alogénico fue de 5 a $16 \%$ antes de la profilaxis. Esta cifra se redujo a 2,5\% con $\mathrm{QP}^{4,5}$. Hasta el momento no hay datos de su incidencia en TPH autólogo.

\section{Factores de riesgo}

\section{Trasplante de órganos sólidos}

Los factores de riesgo para la infección por P. jiroveci en TOS son el uso de FK506, tacrolimus/sirolimus, neuril/
Unidad de Infectología Hospital Barros Luco Trudeau, Santiago. Facultad de Medicina, Universidad de Chile, Santiago (MPG).

Unidad de Infectología Clínica Santa María, Santiago (TB).

Los autores declaran no tener conflictos de interés.

Correspondencia a: pilargambra@yahoo.es tbidart@hotmail.com 
micofenolato, mayor edad, donación expandida, infección concomitante por CMV y rechazo ${ }^{1}$.

\section{Trasplante de precursores hematopoyéticos}

Se han descrito como factores de riesgo en TPH la terapia inmunosupresora persistente, $\mathrm{EICH}$, rechazo, recuento de CD4 $<200 / \mathrm{mm}^{36}$, recaída de patología oncológica y discontinuación precoz de $\mathrm{QP}^{4}$.

\section{Estrategias de profilaxis}

Green y cols. ${ }^{7}$, muestran en un meta-análisis que la QP con cotrimoxazol en pacientes con TOS, TPH autólogo y cáncer hematológico reduce la ocurrencia de $P$. jiroveci en 91\% (RR 0,09 IC 95\% 0,02-0,32) así como también la mortalidad asociada a neumonía por este patógeno. No se logró demostrar disminución de la mortalidad por todas las causas y se estimó que el número de pacientes necesario de tratar para evitar un caso de neumonía por $P$. jiroveci es de 15 . Sólo $3,1 \%$ de los adultos y ningún niño requirió discontinuación de profilaxis ${ }^{7,8}$. Con este estudio quedó establecida la utilidad de la QP con cotrimoxazol y se refuerza el conocimiento empírico hasta ahora existente.

\section{Trasplante de órganos sólidos}

Se han utilizado diferentes esquemas de dosificación con cotrimoxazol en QP de TOS. Dosis alta (160/800 mg/ día -trimetoprim/sulfametoxazol, respectivamente- en régimen diario, en adultos) vs dosis baja $(80 / 400 \mathrm{mg} /$ día en régimen diario, en adultos). Los datos muestran que no existe diferencia entre ambos regímenes. La incidencia en pacientes con dosis altas/bajas de profilaxis fue $0 \%$ $(0 / 181)$ y $1 \%(1 / 105$; rango $0-2 \%)$ respectivamente, por lo que se recomiendan dosis bajas, considerando su menor costo y una mayor tolerabilidad ${ }^{9}$. Se ha evaluado además la aplicación de esquema diario vs tres veces por semana, con resultados de eficacia semejante tanto para TOS como $\mathrm{TPH}^{7}$.

La incidencia de neumonía por $P$. jiroveci en TOS es ocho veces más frecuente durante el primer año post trasplante que en años subsecuentes, lo que no es aplicable en trasplante de pulmón, donde no se observa esta disminución, sugiriendo que en trasplante pulmonar podría ser necesario prolongar la profilaxis por más de un año ${ }^{1}$.

\section{Trasplante de precursores hematopoyéticos}

En pacientes adultos con leucemia linfoblástica aguda (LLA), un estudio retrospectivo analizó la QP con cotrimoxazol indicado tres días consecutivos por semana, siendo tan efectivo como el uso de cotrimoxazol diario ${ }^{10}$. En niños con LLA, no se reportó caso alguno de infección por P. jiroveci, con un esquema profiláctico de cotrimoxazol indicado dos días consecutivos por semana ${ }^{11,12}$.

\section{Alternativas de quimioprofilaxis}

Hasta $40 \%$ de los pacientes adultos sometidos a trasplantes que utilizan QP con cotrimoxazol experimentan reacciones adversas. En estos pacientes se ha analizado y probado otros fármacos alternativos con sabida actividad contra $P$. jiroveci como dapsona, pentamidina en aerosol y atovacuona.

Dapsona es un potente inhibidor de la síntesis de ácido fólico y desde 1980 se ha comunicado su utilidad en prevenir enfermedad por $P$. jiroveci, tanto en pacientes infectados por VIH como en aquellos sometidos a trasplantes; sin embargo, 4 a $13 \%$ de los pacientes con profilaxis con dapsona (100 mg/día) experimentan meta-hemoglobinemia y/o anemia hemolítica. Por otra parte, la intolerancia a cotrimoxazol puede predecir la intolerancia a dapsona. Es posible que los pacientes que experimentan estos eventos adversos tengan una predisposición individual y déficit de glucosa-6-fosfato deshidrogenasa. El metabolismo de dapsona, sirolimus y tacrolimus ocurre en la misma isoenzima del citocromo P-450 (CYP3A4) lo que puede aumentar la incidencia de efectos adversos en pacientes expuestos a estos inmunosupresores $^{13}$. Un estudio caso control mostró un riesgo 18 veces mayor de desarrollar neumonía por $P$. jiroveci en TPH alogénico cuando se realiza profilaxis con dapsona $v s$ cotrimoxazol ${ }^{83}$. Existe escasa publicación acerca del uso de dapsona en niños. Un estudio en niños con LLA y linfoma mostró inferioridad significativa del esquema comparado con cotrimoxazol ${ }^{15}$.

Atovaquona afecta el transporte de electrones en la mitocondria. Los efectos colaterales más comunes son exantema, cefalea, náuseas, diarrea y alteración de las pruebas de función hepática. Generalmente, estos efectos son leves y no requieren discontinuación del fármaco. Tiene actividad contra $P$. jiroveci y $T$. gondii, pero no tiene actividad antibacteriana ${ }^{16}$. La dosis más efectiva es 1.500 $\mathrm{mg}$ /día y sus concentraciones plasmáticas se optimizan si se administra con comidas grasas. En un estudio prospectivo de 25 trasplantes renales, 14 trasplantes hepáticos y 5 trasplantes cardíacos, de receptores intolerantes a cotrimoxazol, los pacientes recibieron profilaxis con atovaquona $1.000 \mathrm{mg} /$ día y ofloxacina $400 \mathrm{mg}$ /día durante seis meses; 39 pacientes completaron la terapia sin complicaciones y no se documentó infecciones por $P$. jiroveci. De los cinco pacientes que discontinuaron la profilaxis antes de los seis meses, dos presentaron intolerancia gástrica y tres receptores de trasplante hepático desarrollaron enfermedad por P. jiroveci $i^{16}$. En un estudio prospectivo, controlado y randomizado, en pacientes sometidos a TPH que recibieron atovacuona $1.500 \mathrm{mg} /$ día con $400 \mathrm{mg}$ de ofloxacina comparado con cotrimoxazol en altas dosis, no se reportó caso alguno de infección por $P$. jiroveci ${ }^{17}$. En TPH, un pequeño estudio randomizado prospectivo 
mostró mejor perfil de tolerabilidad con atovacuona en estos pacientes ${ }^{17,18}$.

Pentamidina aerosolizada o iv en dosis de $300 \mathrm{mg}$ cada 3-4 semanas ha sido reportada en pequeñas series de pacientes que recibieron trasplantes. Fishman mostró que al menos $10 \%$ de los pacientes sometidos a TOS presentaron enfermedad por $P$. jiroveci, lo que hace de éste un medicamento poco eficaz. En general, las neumocistosis se han producido en pacientes con falta de dos o más dosis consecutivas, infección concomitante por CMV, quimioterapia post-trasplante de desordenes linfoproliferativos, uso de globulina anti-linfocitaria y dosis altas de corticoesteroides por rechazo. Las reacciones adversas han sido hipoglicemia, hiperglicemia, tos y broncoespasmo, estas últimas reversibles con terapia broncodilatadora. Al igual que con atovaquona, la administración de pentamidina requiere la co-administración de profilaxis antibacteriana ${ }^{16}$.

La Tabla 2.a muestra los medicamentos y dosis utilizadas en profilaxis de $P$. jiroveci en pacientes sometidos a TOS o TPH.

\section{Resumen}

Pneumocystis jiroveci es un patógeno importante en pacientes sometidos a TOS y TPH. Se recomienda profilaxis universal a todos los pacientes adultos y niños sometidos a TOS o TPH porque su uso reduce significativamente la ocurrencia y mortalidad asociada a neumonía por este agente. El medicamento de elección es cotrimoxazol (A1) tres veces por semana, en dosis bajas, esquema que ha demostrado igual eficacia y mejor tolerancia que el esquema diario y/o con dosis altas. La profilaxis se inicia 7 a 14 días post trasplante en TOS y posterior al implante en TPH, con una duración promedio de 6 meses salvo en trasplante de hígado y pulmón en que se prolonga por 1 año, al igual que en TPH con grado importante de inmunosupresión. Son alternativas de profilaxis dapsona (B2), pentamidina aerosolizada (B2) y atavacuona (C2).
Recomendación $\mathrm{N}^{\circ}$ 2: Profilaxis para neumonía por $P$. jiroveci en trasplante de órganos sólidos y precursores hematopoyéticos

- Se debe administrar a todos los receptores de TOS y TPH (A1)

- Terapia de elección: cotrimoxazol (A1)

Adultos: 80/400 mg/día de trimetoprim/sulfametoxazol, 3 veces/semana en días alternos (A1) Niños: $5 / 20 \mathrm{mg} / \mathrm{kg} /$ día de trimetoprim/sufametoxazol dividido en 2 dosis, tres días consecutivos cada semana (A2)

- Inicio de profilaxis: En TOS día 7-14 post-trasplante, en TPH, luego del implante (C3)

- Duración de la profilaxis: En TOS 6 meses, excepto en trasplante de pulmón e hígado, que se recomienda un año. En TPH, 6 meses post-implante, prolongar en pacientes que persistan con terapia inmunosupresora, EICH crónica, rechazo y LTCD4 < 200/mm³ (B2)

- Alternativa: En caso de reacción adversa grave a cotrimoxazol, se recomienda dapsona por sobre pentamidina aerosolizada (B2). Atavacuona ha mostrado buena tolerancia en pacientes con TPH autólogo (C2)

Tabla 2.a. Dosis de diferentes regímenes para la profilaxis de neumonía por $\boldsymbol{P}$. jiroveci en pacientes sometidos a trasplantes de órganos sólidos o precursores hematopoyéticos

\begin{tabular}{|c|c|c|}
\hline Medicamento & Dosis pediátrica & Dosis para adultos \\
\hline Cotrimoxazol & $\begin{array}{l}\text { 5/20 mg/kg/día de trimetoprim/ } \\
\text { sulfametoxazol repartido en una o } \\
\text { dos tomas diarias } \\
\text { Frecuencia: } \\
2 \text { a } 3 \text { días consecutivos por } \\
\text { semana }\end{array}$ & $\begin{array}{l}80 / 400 \text { mg (dosis baja) o 160/800 } \\
\text { mg/día (dosis alta) de trimetoprim/ } \\
\text { sulfametoxazol } \\
\text { Frecuencia: } \\
3 \text { veces por semana, en días } \\
\text { alternos }\end{array}$ \\
\hline Dapsona & $\begin{array}{l}2 \mathrm{mg} / \mathrm{kg} \text { al día o } 4 \mathrm{mg} / \mathrm{kg} \text { una vez } \\
\text { por semana (> } 1 \text { mes) }\end{array}$ & $\begin{array}{l}100 \text { mg al día o } 200 \text { mg por } \\
\text { semana }\end{array}$ \\
\hline $\begin{array}{l}\text { Pentamidina aerosolizada } \\
\text { ( }>6 \text { años de edad) }\end{array}$ & $\begin{array}{l}300 \text { mg mensualmente vía } \\
\text { respiratoria inhalada }\end{array}$ & $\begin{array}{l}300 \text { mg mensualmente vía } \\
\text { respiratoria inhalada }\end{array}$ \\
\hline $\begin{array}{l}\text { Pentamidina intravenosa } \\
\text { ( } \leq 6 \text { años de edad) }\end{array}$ & $4 \mathrm{mg} / \mathrm{kg}$ al mes & $4 \mathrm{mg} / \mathrm{kg}$ al mes \\
\hline Atovacuona & $\begin{array}{l}30 \mathrm{mg} / \mathrm{kg} \text { una vez al día (máximo } \\
1.500 \mathrm{mg} \text { al día) }\end{array}$ & 1.500 mg al día \\
\hline
\end{tabular}




\section{Referencias bibliográficas}

1.- Gordon S M, LaRosa S P, Kalmadi S, Arroliga A C, Avery R K, Truesdell-LaRosa L, et al. Should prophylaxis for Pneumocystis carinii pneumonia in solid organ transplant recipients ever be discontinued? Clin Infect Dis 1999; 28: 240-6.

2.- Lufft V, Kliem V, Behrend M, Pichlmayr R, Koch KM, Brunkhorst R. Incidence of Pneumocystis carinii pneumonia after renal transplantation. Impact of immunosuppression. Transplantation 1996; 62: 421-3.

3.- Neff R T, Jindal R M, Yoo D Y, Hurst F P, Agodoa L Y, Abbott K C. Analysis of USRDS: incidence and risk factors for Pneumocystis jiroveci pneumonia. Transplantation 2009; 88 : 135-41.

4.- De Castro N, Neuville S, Sarfati C, Ribaud P, Derouin F, Gluckman E, et al. Occurrence of Pneumocystis jiroveci pneumonia after allogeneic stem cell transplantation: a 6-year retrospective study. Bone Marrow Transplant 2005; 36: 879-83.

5.- Rodríiguez M, Fishman J A. Prevention of infection due to Pneumocystis spp. in human immunodeficiency virus-negative immunocompromised patients. Clinical Microbiol Rev 2004; 17: 770-82.

6.- Chen C S, Boeckh M, Seidel K, Clark J G, Kansu E, Madtes DK, et al. Incidence, risk factors, and mortality from pneumonia developing late after hematopoietic stem cell transplantation. Bone Marrow Transplant 2003; 32: 515-22.
7.- Green H, Paul M, Vidal L, Leibovici L. Prophylaxis of Pneumocystis pneumonia in immunocompromised non-HIV-infected patients: systematic review and meta-analysis of randomized controlled trials. Mayo Clinic Proc 2007; 82: 1052-9.

8.- Hughes W T, Kuhn S, Chaudhary S, Feldman S, Verzosa M, Aur R J, et al. Successful chemoprophylaxis for Pneumocystis carinii pneumonitis. New Engl J Med 1977; 297: 1419-26.

9.- Di Cocco P, Orlando G, Bonanni L, D'Angelo M, Clemente K, Greco S, et al. A systematic review of two different trimetoprim-sulfamethoxazole regimens used to prevent Pneumocystis jirovecii and no prophylaxis at all in transplant recipients: appraising the evidence. Transplant Proc 2009; 41: 1201-3.

10.- Hughes W T, Rivera G K, Schell M J, Thornton D, Lott L. Successful intermittent chemoprophylaxis for Pneumocystis carinii pneumonitis. New Engl J Med 1987; 316: 1627-32.

11.- Lindemulder S, Albano E. Successful intermittent prophylaxis with trimethoprim/ sulfamethoxazole 2 days per week for Pneumocystis carinii (jiroveci) pneumonia in pediatric oncology patients. Pediatrics 2007; 120: e47-51.

12.- Agrawal A K, Chang P P, Feusner J. Twice weekly Pneumocystis jiroveci pneumonia prophylaxis with trimethoprimsulfamethoxazole in pediatric patients with acute lymphoblastic leukemia. J Pediatr Hematol Oncol; 33: e1-4.

13.- Lee I, Barton T D, Goral S, Doyle A M, Bloom R D, Chojnowski D, et al. Complications related to dapsone use for Pneumocystis jirovecii pneumonia prophylaxis in solid organ transplant recipients. Am J Transplant 2005; 5: 2791-5.

14.- Souza J P, Boeckh M, Gooley T A, Flowers M E, Crawford S W. High rates of Pneumocystis carinii pneumonia in allogeneic blood and marrow transplant recipients receiving dapsone prophylaxis. Clin Infect Dis 1999; 29: 1467-71.

15.- Prasad P, Nania J J, Shankar S M. Pneumocystis pneumonia in children receiving chemotherapy. Pediatr Blood Cancer 2008; 50: 896-8.

16.- Fishman J A. Prevention of infection caused by Pneumocystis carinii in transplant recipients. Clin Infect Dis 2001; 33: 1397-405.

17.- Colby C, McAfee S, Sackstein R, Finkelstein D, Fishman J, Spitzer T. A prospective randomized trial comparing the toxicity and safety of atovaquone with trimethoprim/sulfamethoxazole as Pneumocystis carinii pneumonia prophylaxis following autologous peripheral blood stem cell transplantation. Bone Marrow Transplant 1999; 24: 897-902.

18.- Madden R M, Pui C H, Hughes W T, Flynn P M, Leung W. Prophylaxis of Pneumocystis carinii pneumonia with atovaquone in children with leukemia. Cancer 2007; 109: 1654-8. 